\title{
Intervention for Prevention of Enthesopathy among Junior High School Soccer Players
}

\author{
Rie NAKAZAWA, RPT, MS ${ }^{1)}$, MasaAKi SAKAmoto, RPT, PhD ${ }^{1)}$, Shigeo MoHara, MD ${ }^{1)}$ \\ 1) Graduate School of Health Sciences, Gunma University: 3-39-15 Showa, Maebashi, \\ Gunma 371-8514, Japan. TEL \& FAX +81-27-220-8972
}

J. Phys. Ther. Sci.
16: $91-99,2004$

\begin{abstract}
The purpose of this study was to examine the transition with time of enthesopathy and the effect of guided stretching, in order to search for the possibility of physical intervention to prevent sports injury during the growth period. The subjects were 81 male soccer players who played in the extramural soccer team. They were divided into two groups: 13 who had experienced enthesopathy problems and a group of 68 who had not. Measurements were taken before and after intervention and were measurements of muscle tightness and alignment, etc. In all subjects, a significant improvement was found in tightness of the bilateral hamstrings and left quadriceps. However, when the results of subjects belonging to the group which had enthesopathy before intervention were compared, significant reinforcement of tightness of the right ilio-psoas was found. The reason for this is considered to be because the intervention method was inadequate. This suggests the importance of individual guidance, and especially confirmation of enforcement and guidance over time for players who have enthesopathy.
\end{abstract}

Key words: Growth period (adolescent), Enthesopathy, Injury prevention

(This article was submitted Mar. 1, 2004, and was accepted May 10, 2004)

\section{INTRODUCTION}

Soccer is a ball sport focusing on the leg. It is a sport in which intense contact occurs, and the frequencies of traumatic injuries and overuse injuries are high ${ }^{1)}$. During the growth period, because there is less intense play with the speed and body contact of the adult sport, the frequency of overuse injuries is higher than traumatic injuries.

In the growth period, long growth and width growth of the bone and the muscle/tendon are not necessarily coincident. At the time of the so-called growth spurt, around 11 years old to 15 years old, although the bone grows quickly, the growth speed of soft tissues such as muscles and tendons is slow. The imbalance of this growth causes stress of extension in the muscle and the tendon. For this reason, it is considered that the possibility of injury in the musculoskeletal junction is high. Koga et al. ${ }^{2)}$ reported that strain of soft tissues, such as the muscle, the tendon, and joint capsule, becomes high, and it has become an item of sports injury which occurs frequently. Furthermore, Torii et al. ${ }^{3)}$ reported that there was a relationship between the occurrence of athletic injury and joint laxity/muscle tightness of adolescent athletes in junior high school and high school. Moreover, Okamura et al. ${ }^{4)}$ reported that alignment check is more useful as a medical check of injury among school children and junior high school students in comparison with senior high school students. For this reason, the authors thought that muscle tightness and leg alignment were greatly involved in the occurrence of enthesopathy, in which mechanical stimulation to maintain the musculoskeletal junction causes pain at rest and in motion. Accordingly we have studied factors related to the occurrence of enthesopathy in junior high school soccer players. The results reveal that discriminant analysis significantly differentiated between the players with 
Table 1. Subjects' attributes

\begin{tabular}{lcccc}
\hline & third graders & second graders & first graders & total \\
\hline number & 28 & 21 & 32 & 81 \\
average height \pm SD $(\mathrm{cm})$ & $166.2 \pm 6.1$ & $158.2 \pm 7.3$ & $149.5 \pm 6.2$ & $157.5 \pm 9.6$ \\
average weight \pm SD $(\mathrm{kg})$ & $56.7 \pm 9.6$ & $49.3 \pm 8.9$ & $43.8 \pm 7.2$ & $49.7 \pm 10.1$ \\
average body fat ratio \pm SD (\%) & $18.5 \pm 5.6$ & $16.4 \pm 4.5$ & $19.3 \pm 5.4$ & $18.3 \pm 5.3$ \\
soccer start age \pm SD (year) & $8.2 \pm 1.7$ & $8.6 \pm 1.8$ & $8.4 \pm 1.8$ & $8.4 \pm 1.8$ \\
team of participation \pm SD (year) & $6.8 \pm 1.7$ & $5.4 \pm 1.8$ & $4.6 \pm 1.8$ & $5.6 \pm 2.0$ \\
\hline
\end{tabular}

SD: standard deviation.

enthesopathy and the players who had not. It was clear that muscle tightness and leg alignment participated in the occurrence of enthesopathy. The purpose of this study was to examine the transition with time of enthesopathy and the effect of intervention by stretching on junior high school soccer players, in order to search for the possibility of physical intervention for prevention of sports injuries during the growth period.

\section{MATERIALS AND METHODS}

\section{Subjects}

The subjects were 81 male soccer players who played on the extramural soccer teams of three junior high schools (mean age $13.1 \pm 1.0$ years). The subjects according to grade were 28 third graders, 21 second graders, and 32 first graders. They had a mean body height of $157.5 \pm 9.6 \mathrm{~cm}$, mean body weight of $49.7 \pm 10.1 \mathrm{~kg}$, mean body fat ratio of $18.3 \pm 5.3 \%$, mean soccer starting age of 8.4 \pm 1.8 years, and mean of team participation of $5.6 \pm$ 2.0 years (Table 1$)$. Moreover, their practice time per day was an average of 2 hours, and the practice frequency was 6 days a week in all three schools. For pivot legs, the left was 76 students and the right was 5 students.

\section{Methods}

Investigations and measurements were performed before the start of soccer practice on all subjects. In addition, the purpose of this study was fully explained to the schools' principals and coaches, and after obtaining consent, investigations and measurements were performed.

\section{1) Investigation items}

Palpation of parts with pain of motion and tenderness of musculoskeletal junction was performed, on subjects who had some pain, with oral consultation in order to check the existence of enthesopathy. Furthermore, a questionnaire about soccer activity which asked about soccer start age (team of participation) and pivot leg was performed.

\section{2) Measurement items}

Measurements of muscle tightness were performed with reference to the tightness test of Torii et al. ${ }^{5)}$ on bilateral ilio-psoas, quadriceps, hamstrings, and gastrocnemius. The joint angle was measured using goniometry. Furthermore, hip abduction joint angle in the standing position (hip adductors) was also measured (Fig. 1).

Measurements of leg alignment were performed on the bilateral Q-angle and leg-heel angle (LHA) in the standing position (Fig. 2). The Q-angle is the angle made between the patella tendon and the line which connects the anterior superior iliac spine to the patella middle point. The LHA is the angle made between the long-axis line of the distal $1 / 3$ part of the leg and the vertical-axis line of the calcaneus. The LHA were expressed so that the direction of pronation of the calcaneus was positive, and the direction of supination of the calcaneus was negative.

The joint laxity test tested seven items according to the generalized joint laxity test of the University of Tokyo ${ }^{5)}$ (Fig. 3), and the total of positive items was taken as the measured value.

\section{3) Intervention method}

A feedback paper, on which stretching methods and the icing method were written, was made for the purpose of the improvement of enthesopathy and injury prevention (Fig. 4). Individual guidance for all subjects and feedback to coaches was also performed. The same investigations and measurements were performed again after three months. Furthermore, questionnaires about the enforcement of the guidance contents, such as 


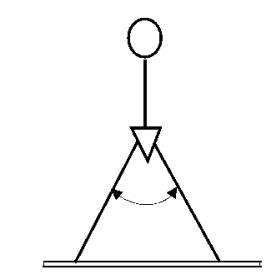

hip adductors

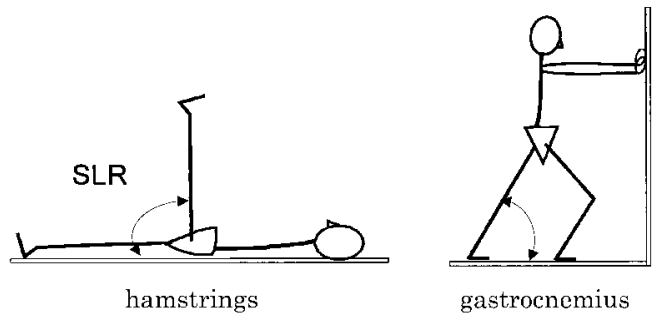

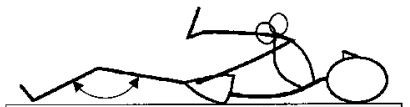

ilio-psoas

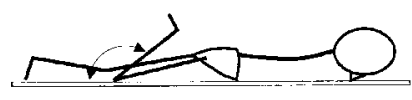

quadriceps

Fig. 1. Tightness test (adapted from 5).

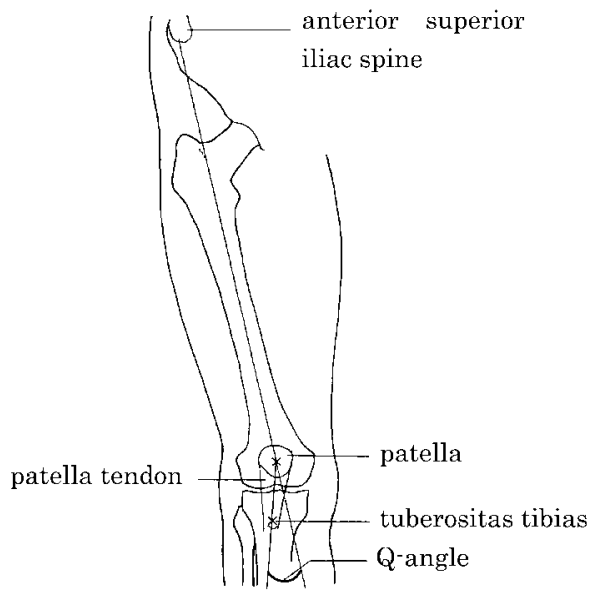

Q-angle

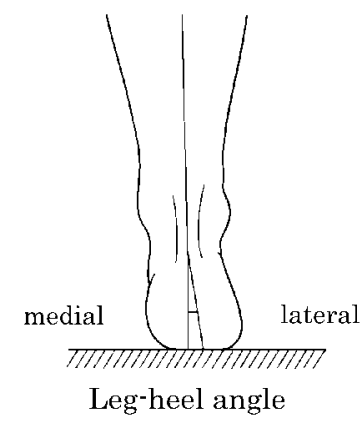

Fig. 2. Alignment test

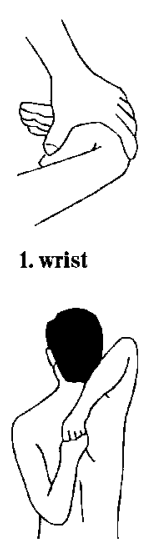

5. shoulder
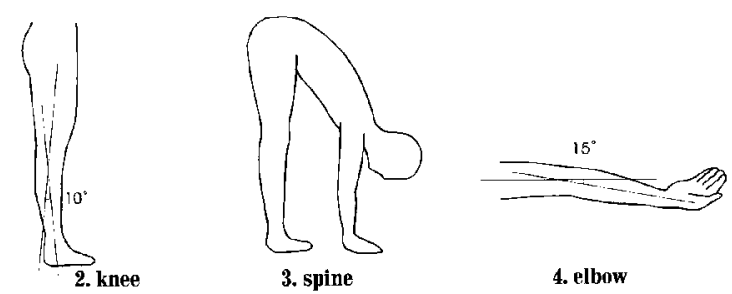

Fig. 3. Generalized joint laxity test ${ }^{5)}$. 
To

We report the results of your measurements. The following $V$ marks items which are points that you had better pay attention to for prevention of the injury. As a prevention method, stretching before and after practice is recommended. For stretching exercises refer to pictures 1-18. For you, an especially important stretching exercise is attached o. Please also perform at home. (It is very effective after a bath!)

$\square$ The muscles of the anterior are shortening

$\square$ The muscles of the medial are shortening

$\square$ The muscles of the calf are shortening

Since V mark has been made to the above, you need icing after practice, or already have injuries. Please perform icing by the following methods.

$\square$ icing recommended.

<icing method>

The cold pack (something like ice) or vinyl bag containing ice is put on the part with pain. The cold pack is wrapped in a towel. Although you may at first feel cold and pain, you will gradually get used to it over 4 to 5 minutes. After that, if you don't feel cold and pain, you should perform icing for 15 to 30 minutes. However, if you lose feeling, you should stop cooling at once. (Frostbite may have started.) If you have feeling, you should keep on cooling as long as possible.

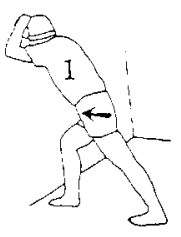

each leg $20 \mathrm{sec}$

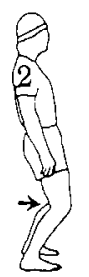

$30 \mathrm{sec}$

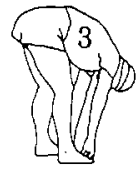

$20 \mathrm{sec}$

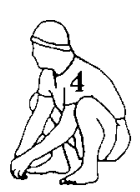

$20 \mathrm{sec}$

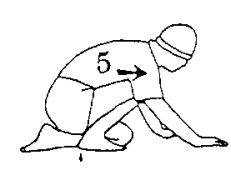

each leg $20 \mathrm{sec}$

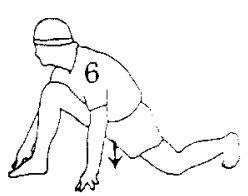

each leg $20 \mathrm{sec}$

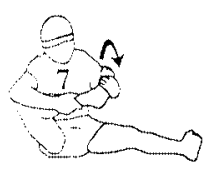

each direction 10 times

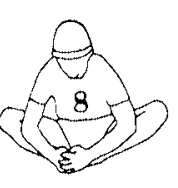

$30 \mathrm{sec}$

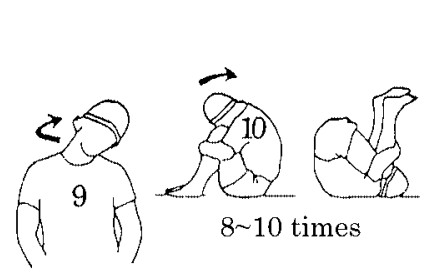

each direction 5 times
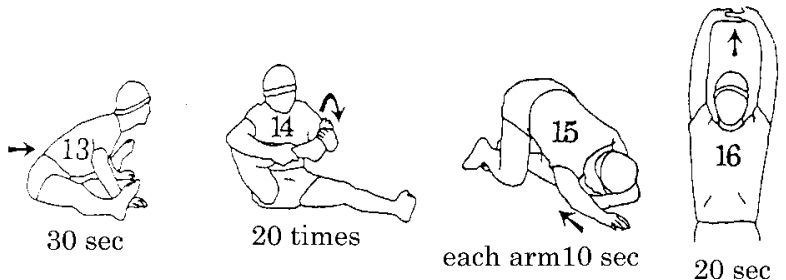

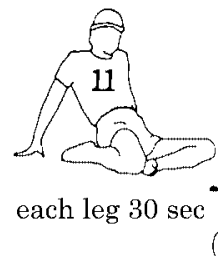

left and right $10 \mathrm{sec}$

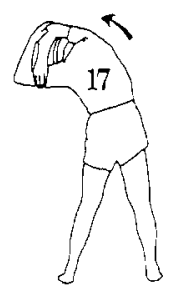

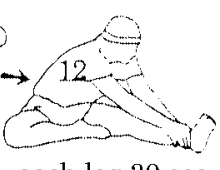

each leg $30 \mathrm{sec}$

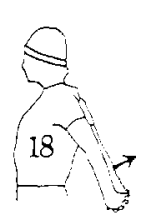

$20 \mathrm{sec}$

Fig. 4. Feedback paper. 
Table 2. The number of injuries and enthesopathy before and after intervention

\begin{tabular}{|c|c|c|}
\hline & before $(n=81)$ & after $(n=73)$ \\
\hline injury number & $24(30)$ & $28(38)$ \\
\hline part: upper extremity & $0(0)$ & $3(8)$ \\
\hline lower extremity & $38(93)$ & $35(87)$ \\
\hline trunk & $4(7)$ & $2(5)$ \\
\hline total & $42(100)$ & $40(100)$ \\
\hline enthesopathy number & $13(16)$ & $10(14)$ \\
\hline part: patella inferior & $3(14)$ & $1(6)$ \\
\hline patella tendon & $4(19)$ & $5(28)$ \\
\hline tuberositas tibias & $10(48)$ & $8(44)$ \\
\hline Achilles tendon & $4(19)$ & $4(22)$ \\
\hline total & $21(100)$ & $18(100)$ \\
\hline
\end{tabular}

frequency and time of stretching, were also performed at the same time.

\section{4) Analysis method}

Correlation analysis was used for all investigation and measurement items in order to examine relationships among items. The subjects were divided into two groups: the group with those who had experienced enthesopathy problems (group A), and the group with those who had not (group B). Then, the non-paired t-test between the 2 groups was used for each item.

In order to examine the effect of intervention, the paired t-test before and after intervention in all subjects was used for each item. Furthermore, the paired t-test before and after intervention in every group was used.

\section{RESULTS}

\section{The frequency of injury before intervention}

At the time of the first investigation, the number of those who had pain was $24(30 \%)$, and items of pain totaled 42 . There were $38(93 \%)$ items of pain of the lower extremity, and $4(7 \%)$ items of trunk pain. No pain of the upper extremity was reported. In pain of the lower extremity, most subjects experienced pain of the musculoskeletal junction around the knee and the ankle. The number of those subjects was $13(16 \%), 21$ items $(55 \%)$. As for the location of pain, there were $3(14 \%)$ items of the patella inferior, $4(19 \%)$ items of the patella tendon, $10(48 \%)$ items of the tuberositas tibias, and 4 $(19 \%)$ items of the Achilles tendon parts (Table 2). As mentioned above, group A had 13 persons and group B had 68 persons.

\section{Result before intervention}

The results of correlation analysis to all the investigation and measurement items revealed that the correlation of the right and left was high and the correlation of each factor was low. Moreover, a significant difference was observed only for the right ilio-psoas in the non-paired t-test between the

Table 3. The non-paired t-test between group A $(n=13)$ and group $B(n=68)$

\begin{tabular}{lccc}
\hline & group A & group B & P \\
\hline team of participation (year) & $6.1 \pm 2.0$ & $5.5 \pm 2.0$ & N.S \\
tightness $\left(^{\circ}\right)$ & & & \\
R ilio-psoas & $10.4 \pm 7.5$ & $14.3 \pm 6.9$ & $*$ \\
L ilio-psoas & $11.5 \pm 6.6$ & $13.0 \pm 6.0$ & N.S \\
R hamstrings & $77.3 \pm 14.2$ & $71.0 \pm 10.6$ & N.S \\
L hamstrings & $78.5 \pm 15.1$ & $73.5 \pm 10.0$ & N.S \\
R quadriceps & $135.4 \pm 12.3$ & $134.9 \pm 10.3$ & N.S \\
L quadriceps & $134.2 \pm 12.4$ & $136.3 \pm 10.1$ & N.S \\
R gastrocnemius & $25.0 \pm 8.9$ & $23.9 \pm 6.5$ & N.S \\
L gastrocnemius & $24.6 \pm 9.0$ & $22.9 \pm 5.7$ & N.S \\
hip adductors & $120.0 \pm 12.8$ & $123.2 \pm 11.5$ & N.S \\
joint laxity & $2.2 \pm 1.6$ & $2.0 \pm 1.2$ & N.S \\
alignment $\left({ }^{\circ}\right)$ & & & \\
R Q-angle & $10.0 \pm 1.6$ & $10.1 \pm 2.3$ & N.S \\
L Q-angle & $10.2 \pm 1.8$ & $10.1 \pm 2.4$ & N.S \\
R LHA & $4.2 \pm 1.5$ & $4.0 \pm 2.6$ & N.S \\
L LHA & $4.0 \pm 1.9$ & $3.7 \pm 2.5$ & N.S \\
\hline
\end{tabular}

Mean \pm S.D., N.S: not significant, *:p $<0.05$. 
Table 4. The paired t-test before and after intervention for each item in all subjects $(n=73)$

\begin{tabular}{lccc}
\hline & before & after & P \\
\hline tightness $\left(^{\circ}\right)$ & & & \\
R ilio-psoas & $13.7 \pm 7.1$ & $13.7 \pm 6.4$ & N.S \\
L ilio-psoas & $12.8 \pm 6.1$ & $12.1 \pm 4.6$ & N.S \\
R hamstrings & $72.0 \pm 11.4$ & $78.8 \pm 10.1$ & $* * *$ \\
L hamstrings & $74.3 \pm 11.0$ & $79.3 \pm 10.2$ & $* * *$ \\
R quadriceps & $134.9 \pm 10.6$ & $136.2 \pm 10.0$ & N.S \\
L quadriceps & $135.9 \pm 10.5$ & $139.8 \pm 8.4$ & $* * *$ \\
R gastrocnemius & $24.1 \pm 6.9$ & $24.5 \pm 6.2$ & N.S \\
L gastrocnemius & $23.2 \pm 6.3$ & $24.6 \pm 6.6$ & N.S \\
hip adductors & $122.7 \pm 11.7$ & $121.9 \pm 11.1$ & N.S \\
joint laxity & $2.1 \pm 1.3$ & $2.3 \pm 1.1$ & $* *$ \\
alignment $\left({ }^{\circ}\right)$ & & & \\
R Q-angle & $10.1 \pm 2.2$ & $10.5 \pm 2.4$ & N.S \\
L Q-angle & $10.2 \pm 2.3$ & $10.8 \pm 2.9$ & N.S \\
R LHA & $4.0 \pm 2.5$ & $4.6 \pm 3.5$ & N.S \\
L LHA & $3.7 \pm 2.4$ & $5.5 \pm 3.7$ & $* * *$ \\
\hline
\end{tabular}

Mean \pm S.D., N.S: not significant, ${ }^{* *}: \mathrm{p}<0.01,{ }^{* * *}: \mathrm{p}<0.001$.

two groups $(\mathrm{p}<0.05)($ Table 3$)$.

\section{The frequency of the injury after intervention}

The number of those who had pain was $28(38 \%)$ among 73 subjects ( 25 third graders, 20 second graders, and 28 first graders) for whom measurement was possible after intervention, and items of pain totaled 40. Pain of the lower extremity was most, 35 items $(87 \%)$. Items of upper extremity pain were $3(8 \%)$, and items of trunk pain were 2 $(5 \%)$. The number of the persons with enthesopathy were $10(14 \%), 18$ items $(45 \%)$. The location of pain was as follows: the patella inferior, $1(6 \%)$; the patella tendon, 5 (28\%); the tuberositas tibias, 8 (44\%); and the Achilles tendon, 4 (22\%) (Table 2). There were two subjects who belonged to group A before intervention who had changed to group B after intervention, and there was one subject who belonged to group B before intervention who had changed to group A after intervention.

\section{Result after intervention}

The result of the paired t-test before and after intervention for each item in all subjects showed a significant difference in five items: tightness of bilateral hamstrings, left quadriceps, joint laxity, and left LHA $(\mathrm{p}<0.05)$ (Table 4). For the 11 persons who had belonged to group A before intervention, and for whom measurement was possible after intervention, a significant difference was observed in tightness of right ilio-psoas and bilateral LHA $(p<0.05)$ (Table 5). Moreover, for 62 persons who belonged to group B before intervention and for whom measurement was possible after intervention, a significant difference was observed in tightness of bilateral hamstrings, left quadriceps and left LHA $(\mathrm{p}<0.05)$ (Table 6).

\section{Enforcement situation of the stretching intervention}

The number of the schools which performed stretching in soccer practice each time was two, and one school was not performing stretching after feedback. There were 45 subjects $(62 \%)$ who were performing the stretching exercises as independent training among the 73 subjects for whom measurement after intervention was possible. The average frequency of the stretching exercises was $2.9 \pm 1.9$ times, and the average time was $7.8 \pm 4.9$ minutes per time. Among the 11 persons who belonged to group A before intervention, and for whom measurement was possible after intervention, the number of subjects who were performing stretching exercises independently was $9(82 \%)$. Their average frequency was $3.3 \pm 2.0$ times, and their average time was $12.8 \pm 5.7$ minutes per time. Furthermore, among the 62 persons who belonged to B group before intervention and for whom measurement was possible after intervention, the number of subjects who were performing stretching exercises independently was $36(50 \%)$. Their 
Table 5. The paired t-test before and after intervention for each item in A group $(n=11)$

\begin{tabular}{lccc}
\hline & before & after & $\mathrm{P}$ \\
\hline tightness $\left(^{\circ}\right)$ & & & \\
R ilio-psoas & $10.5 \pm 8.2$ & $15.5 \pm 4.7$ & $* *$ \\
L ilio-psoas & $11.4 \pm 7.1$ & $10.9 \pm 3.8$ & N.S \\
R hamstrings & $78.6 \pm 15.2$ & $81.4 \pm 10.5$ & N.S \\
L hamstrings & $79.6 \pm 16.2$ & $81.8 \pm 11.6$ & N.S \\
R quadriceps & $138.2 \pm 11.0$ & $140.9 \pm 10.2$ & N.S \\
L quadriceps & $137.3 \pm 10.8$ & $142.3 \pm 9.3$ & N.S \\
R gastrocnemius & $25.5 \pm 9.1$ & $26.8 \pm 8.5$ & N.S \\
L gastrocnemius & $25.9 \pm 8.6$ & $26.4 \pm 9.2$ & N.S \\
hip adductors & $118.6 \pm 13.4$ & $126.8 \pm 14.2$ & N.S \\
joint laxity & $2.3 \pm 1.8$ & $2.3 \pm 1.5$ & N.S \\
alignment $\left({ }^{\circ}\right)$ & & & \\
R Q-angle & $10.0 \pm 1.7$ & $11.5 \pm 2.8$ & N.S \\
L Q-angle & $10.5 \pm 1.9$ & $12.2 \pm 2.8$ & N.S \\
R LHA & $4.1 \pm 1.5$ & $6.2 \pm 2.9$ & $* *$ \\
L LHA & $4.0 \pm 2.1$ & $7.5 \pm 4.1$ & $* *$ \\
\hline
\end{tabular}

Mean \pm S.D., N.S: not significant, **: $\mathrm{p}<0.01$.

Table 6. The paired t-test before and after intervention for each items in B group $(n=62)$

\begin{tabular}{lccc}
\hline & before & after & P \\
\hline tightness $\left(^{\circ}\right)$ & & & \\
R ilio-psoas & $14.5 \pm 6.9$ & $13.4 \pm 6.6$ & N.S \\
L ilio-psoas & $13.1 \pm 6.1$ & $12.3 \pm 4.7$ & N.S \\
R hamstrings & $71.6 \pm 9.6$ & $78.3 \pm 10.0$ & $* * *$ \\
L hamstrings & $74.2 \pm 9.7$ & $78.9 \pm 9.9$ & $* * *$ \\
R quadriceps & $134.6 \pm 10.1$ & $135.3 \pm 9.8$ & N.S \\
L quadriceps & $135.6 \pm 10.2$ & $139.4 \pm 8.2$ & $* * *$ \\
R gastrocnemius & $24.1 \pm 6.4$ & $24.0 \pm 5.7$ & N.S \\
L gastrocnemius & $22.9 \pm 5.9$ & $24.3 \pm 6.1$ & N.S \\
hip adductors & $123.5 \pm 11.1$ & $121.1 \pm 10.4$ & N.S \\
joint laxity & $2.0 \pm 1.2$ & $2.4 \pm 1.1$ & $* *$ \\
alignment $\left({ }^{\circ}\right)$ & & & \\
R Q-angle & $10.2 \pm 2.3$ & $10.3 \pm 2.3$ & N.S \\
L Q-angle & $10.2 \pm 2.4$ & $10.5 \pm 2.9$ & N.S \\
R LHA & $4.0 \pm 2.5$ & $4.4 \pm 3.6$ & N.S \\
L LHA & $3.7 \pm 2.5$ & $5.1 \pm 3.6$ & $* *$ \\
\hline
\end{tabular}

Mean \pm S.D., N.S: not significant, ${ }^{* *}: \mathrm{p}<0.01, * * *: \mathrm{p}<0.001$.

average frequency was $2.8 \pm 1.9$ times, their average time was $6.6 \pm 3.9$ minutes per time, and their training time was significantly shorter than group A.

\section{DISCUSSION}

\section{The frequency and location of injury}

For location of soccer injury during the growth period, Ikeda et al. ${ }^{6)}$ reported that injury of the lower extremity was $77 \%$ of the whole among junior youth players. Furthermore, Sakamoto et al. ${ }^{1)}$ reported that injury of the lower extremity was $90 \%$ of the whole among high school soccer players. The result of this study was that pain of the lower extremity was $93 \%$ of the whole and it showed the same trend as the other two reports. Soccer is a sport mainly concerned with ball control 
by the lower extremity. For this reason, it is considered that injuries of the lower extremity occur easily irrespective of the game level.

\section{Tightness in the players with enthesopathy}

Tightness of right ilio-psoas was significantly high in the players with enthesopathy. Torii et al. $\left.{ }^{4}\right)$ reported that tightness of ilio-psoas was significantly high in junior high school players and male high school students with injury, as a result of examining the relationship between athletic injury and muscle tightness. This study showed almost the same trend as the report of Torii et al. ${ }^{4)}$. Among junior high school soccer players, it is considered to be especially necessary to pay attention to tightness of ilio-psoas for players with enthesopathy.

\section{Intervention for the junior high school soccer players}

Intervention, stretching etc, for the purpose of improvement of pain and injury prevention was performed, and the transition with time of the pain was examined and the effect was judged. After intervention, there were two subjects whose pain of musculoskeletal junction disappeared, and there was one subject whose pain of musculoskeletal junction appeared. Pain as a percentage of all subjects, was not found to differ, in the number of the persons with pain before and after intervention. As compared with before intervention, a significant improvement was found in tightness of bilateral hamstrings and left quadriceps after intervention. This was considered to be the effect of stretching. However, when only subjects who belonged to group A before intervention were compared, a significant improvement was not found in tightness, but a significant increase was found in tightness of the right ilio-psoas. When subjects who belonged to group B before intervention were compared, an improvement trend was seen in tightness of the bilateral ilio-psoas although it was not significant. Because group A was performing stretching independently with higher frequency than group B, it was shown that consciousness of the necessity for stretching and pain improvement in group A was higher than in group B. Therefore, it is thought that there was a problem with the guidance for the stretching method. Yamamoto et al. ${ }^{7)}$ reported that there were many players who didn't understand the correct method of stretching, as a result of performing a questionnaire about stretching among university sports players. The subjects of this study were junior high school students. Therefore we consider that they were not able to understand the correct method of stretching. We question whether or not the ilio-psoas stretching was performed correctly, because the method of the ilio-psoas stretching is especially difficult. We consider that individual guidance is important and confirmation of especially whether the target muscle stretching is being performed by the player who has an injury. Additionally, education for understanding of the correct method of stretching is necessary.

Moreover, there were two schools which were performing stretching in soccer practice, and one school which was not. Also, the latter was not performing stretching even after feedback. It is considered that this showed that consciousness of injury prevention was low among the coaches. We consider that it is important to pay attention to the consciousness of injury and the necessity of stretching and icing for injury prevention to the players, as well as re-examination of the methods of guidance, such as the confirmation of enforcement and guidance contents with time.

\section{CONCLUSION}

The purpose of this study was to examine the transition with time of enthesopathy and the effect of intervention by guided stretching among 81 male junior high school soccer players. Before intervention, tightness of right ilio-psoas was significantly high in the players with enthesopathy. After intervention, tightness of right ilio-psoas was significantly increased in the players with enthesopathy. This suggests the importance of individual guidance and confirmation of enforcement and guidance contents with time to the player who has an injury.

\section{ACKNOWLEDGMENTS}

The authors thank the principals, coaches, and soccer members of each junior high school which cooperated with this study.

\section{REFERENCES}

1) Sakamoto M, Masunaga M, Nara T, et al.: Soccer injuries in elite high school players. Journal of Athletic Rehabilitation, 1998, 1: 17-20. 
2) Koga $Y$, Omori G, Tanabe $Y$, et al.: Sports disorders in growth period. Orthop Surg Traumatol, 2000, 43: 1181-1187.

3) Torii S: Orthopedic check-up for prevention of athletic injury in adolescency (second report) - prospective study about the relationship between joint laxity, muscle tightness and athletic injury. Jpn J Orthop Sports Med, 1995, 14: 359-366.

4) Okamura Y, Harata S, Mochizuki M, et al.: The evaluation of orthopaedic medical check for sports injury in adolescent athletes. J East Jpn Orthop Traumatol, 1999, 11: 506-509.

5) Torii S: Management and prevention for injuries of adolescent athletes in track and field. Orthop Surg Traumatol, 2000, 43: 1311-1318.

6) Ikeda H, Kurosawa H, Sakuraba K, et al.: Sports injuries of juvenile soccer players. J East Jpn Orthop Traumatol, 1999, 11: 18-21.

7) Yamamoto $T$ : Sports and stretching. Journal of Physical Therapy, 1990, 7: 351-361. 